\title{
Evaluation of the cost-effectiveness of evolocumab in the FOURIER study: a Canadian analysis
}

\author{
Todd C. Lee MD MPH, Mohammed Kaouache PhD, Steven A. Grover MD MPA
}

\section{Abstract}

Background: Evolocumab, a proprotein convertase subtilisin-kexin type 9 (PCSK9) inhibitor, has been shown to reduce low-density lipoprotein levels by up to $60 \%$. Despite the absence of a reduction in overall or cardiovascular mortality in the Further Cardiovascular Outcomes Research With PCSK9 Inhibition in Subjects With Elevated Risk (FOURIER) trial, some believe that, with longer treatment, such a benefit might eventually be realized. Our aim was to estimate the potential mortality benefit over a patient's lifetime and the cost per year of life saved (YOLS) for an average Canadian with established coronary artery disease. We also sought to estimate the price threshold at which evolocumab might be considered cost-effective for secondary prevention in Canada.

Methods: We calibrated the Cardio-metabolic Model, a well-validated tool for predicting cardiovascular events and life expectancy, to the reduction in nonfatal events seen in the FOURIER trial. Assuming that long-term treatment will eventually result in mortality benefits, we estimated YOLSs and cost per YOLS with evolocumab treatment plus a statin compared to a statin alone. We then estimated the annual drug costs that would provide a $50 \%$ chance of being cost-effective at willingness-to-pay values of $\$ 50000$ and $\$ 100000$.

Results: In secondary prevention in patients similar to those in the FOURIER study, evolocumab treatment would save an average of 0.34 (95\% confidence interval [Cl] 0.27-0.41) life-years at a cost of $\$ 101899$ (95\% Cl $\$ 97325-\$ 106473)$, yielding a cost per YOLS of $\$ 299482$. We estimate that to have a 50\% probability of achieving a cost per YOLS below $\$ 50000$ and $\$ 100000$ would require annual drug costs below $\$ 1200$ and $\$ 2300$, respectively.

Interpretation: At current pricing, the use of evolocumab for secondary prevention is unlikely to be cost-effective in Canada.

I schemic heart disease is common, with an estimated 1 in 12 Canadians older than age 20 years affected and more than 150000 new cases in Canada in 2012-2013. ${ }^{1}$ There have been tremendous improvements in the care of patients with cardiovascular disease brought about by therapeutic advances in risk factor modification including cholesterol lowering with statins and technical advances in cardiac critical care. Consequently, since 2000, the rate of death from ischemic heart disease has fallen in Canada by $23 \% .^{1}$ Given these improvements in care, it has become increasingly challenging for newer therapies to have as large an impact on mortality as seen with previous innovations.

Nevertheless, there remains great interest in new therapies to further decrease attributable morbidity and mortality. Following the success of the statins, several other medications that lower low-density lipoprotein (LDL) levels have been investigated for their ability to affect cardiovascular outcomes. The proprotein convertase subtilisin-kexin type 9 (PCSK9) inhibitors, including evolocumab, have garnered interest owing to their ability to lower LDL levels by up to $60 \% .^{2}$
This LDL lowering comes at an estimated cost approaching $\$ 7500$ per year. Until recently, the impact of these drugs on hard outcomes remained unclear.

With the publication of the Further Cardiovascular Outcomes Research With PCSK9 Inhibition in Subjects With Elevated Risk (FOURIER) trial, ${ }^{2}$ in March 2017, we now have evidence that these agents can reduce some cardiovascular outcomes in secondary prevention in patients who are already taking a statin. In this randomized double-blind placebo-controlled trial involving 27564 patients with preexisting atherosclerotic cardiovascular heart disease, the addition of evolocumab reduced the risk of the primary outcome

\section{Competing interests: None declared.}

This article has been peer reviewed.

Correspondence to: Todd Lee, todd.lee@mcgill.ca

CMAJ Open 2018. DOI:10.9778/cmajo.20180011 
(composite of cardiovascular death, myocardial infarction, stroke, hospital admission for unstable angina or coronary revascularization) from $11.3 \%$ to $9.8 \%$ after a median of 2.2 years. Despite the absence of a reduction in overall or cardiovascular mortality in this trial, some believe that, with longer treatment, such a benefit might eventually be realized. ${ }^{3}$ This would also provide the primary justification for prescribing this additional treatment to patients who already have cardiovascular disease. We therefore sought to estimate the potential mortality benefit over a patient's lifetime using an existing well-validated disease-simulation approach that incorporates the Cardiovascular Disease Life Expectancy Model ${ }^{4}$ and furthermore to estimate the cost per year of life saved (YOLS) for an average Canadian with established coronary artery disease. We also sought to estimate the price threshold at which evolocumab might be considered cost-effective for secondary prevention in Canada.

\section{Methods}

\section{Model overview}

The Cardio-metabolic Model was designed to estimate the clinical impact of multiple risk factors for diabetes and cardiovascular disease on life expectancy. ${ }^{5}$ These risk factors include age, sex, blood pressure, total cholesterol level, level of high-density lipoprotein cholesterol and other important factors. ${ }^{5}$ These cardiovascular risk factors in turn increase the risk of dying from coronary disease or cerebrovascular disease. The Cardio-metabolic Model includes the Cardiovascular Life Expectancy Model to estimate the risk of development of fatal or nonfatal coronary disease or cerebrovascular disease, and/or the risk of dying from a noncardiovascular cause. Further details of the Cardio-metabolic Model can be found in previously published supplementary material. ${ }^{5}$

\section{Cardiovascular Life Expectancy Model}

This Markov model has been described in detail previously. ${ }^{4-9}$ Briefly, the model is based on data from the $15 \%$ random sample of the Lipid Research Clinics cohort. ${ }^{4}$ The model estimates the annual probability of dying from coronary heart disease, stroke or other causes. Initially, the model was validated on the fatal outcomes observed in published lipid and hypertension clinical trials. ${ }^{4}$ Similarly, the cardiovascular outcomes for people with diabetes were validated from trial results. ${ }^{9}$ The life expectancy estimates generated by the model have also been validated against published life tables for Americans and Canadians. ${ }^{6,7}$ The model was developed with the use of SAS version 9.3 (SAS Institute). Patients are followed until they die or reach the age of 102 years, at which time they are assumed to have died.

Once we originally validated the model estimates on fatal outcomes, we estimated the risk of nonfatal cardiovascular disease outcomes as follows. We estimated the probabilities of coronary insufficiency, nonfatal myocardial infarction, a transient ischemic attack or a nonfatal stroke by the ratios of nonfatal to fatal events predicted by the results of several studies providing primary or secondary cardiovascular disease outcomes. ${ }^{10-15}$ We subsequently also estimated the probability of various vascular procedures using data on hospital admissions and use of health care services from Canada and the United States. ${ }^{8}$

\section{Calibration of model to estimate results of FOURIER study}

For this study, we calibrated the Cardio-metabolic Model so that its results would approximate those observed in the FOURIER trial in terms of the hazard ratio for the combined outcome (cardiovascular death, myocardial infarction or stroke) over a 2-year follow-up period. We identified lower and upper bounds on our LDL coefficients that produced hazard ratios similar to the confidence intervals (CIs) for the hazard ratio in the FOURIER trial. We then incorporated uncertainty about the LDL coefficients by eliciting normal distributions with standard deviations equal to one-quarter the difference between the upper and lower bounds.

Cardiovascular mortality and total mortality were similar between the treatment and control groups in the FOURIER study despite the observed reduction in nonfatal cardiovascular events. This may have been because evolocumab has no effect on mortality in this population, in which case it would be impossible to show the cost-effectiveness for preventing death. We therefore assumed, given the reduction of nonfatal cardiovascular events by about $20 \%-25 \%$ with evolocumab, a proportional reduction in fatal events that would follow that seen for statins. ${ }^{8}$

\section{Estimation of years of life saved and cost-effectiveness of lipid modification}

Our modelling approach has been described in detail elsewhere. ${ }^{4}$ When comparing treatments having a differential effect on risk factors, the benefits associated with 1 treatment over the other are calculated as the YOLS due to the "first" treatment over the "second" treatment. This value is computed as $\mathrm{YOLS}=\mathrm{LE}_{\text {first }}-\mathrm{LE}_{\text {second, }}$, where LE indicates life expectancy. In the current study, the first treatment was receipt of evolocumab in addition to a statin, and the second treatment was receipt of a statin alone.

The methods used to assign unit cost to acute events and long-term treatments have also been previously described. ${ }^{8}$ In the current analysis, we inflated previously estimated treatment costs to 2017 costs using the Canadian Health Care Inflation Index published by Statistics Canada. The cost of evolocumab was based on the expected Canadian market price $^{16}$ rounded up to $\$ 7500$ annually to account for unmeasured costs of distribution and administration. Uncertainty about our estimated costs was expressed by eliciting uniform distributions on a range of values (point estimates $\pm 20 \%$ ).

We calculated the incremental costs of treatment per YOLS by calculating the difference between lifetime medical costs with and without evolocumab, divided by the difference in the forecasted life expectancies. Because the costs and the health outcomes occur at different times, we discounted both by $3 \%$ annually so as to be comparable to the 
literature. ${ }^{17}$ We also performed a sensitivity analysis at a discounting rate of $1.5 \%$, in keeping with recent Canadian recommendations. ${ }^{18}$

Simultaneously adjusting for uncertainty in costs and model coefficients involved simulating 1000 sets of values for these coefficients and costs from their respective distributions. For each simulated set of values, we obtained an estimate of YOLSs and lifetime incremental costs. Using these results of YOLSs and incremental costs, we constructed CIs around our estimates. We then generated cost-effectiveness acceptability curves showing, as a function of the annual drug cost, the proportion of the 1000 simulated results that would give, for a specified willingness-to-pay amount, a positive incremental net benefit.

\section{Results}

The estimated numbers of events prevented with therapy are presented in Table 1, and the estimated costs of cardiovascular or other events are included in Supplementary Table S1, Appendix 1 (available at www.cmajopen.ca/content/6/2/E162/ suppl/DC1). Despite no reduction in cardiovascular deaths observed in the FOURIER trial, the model assumes that, over a patient's lifetime, the positive impact on LDL level and nonfatal myocardial infarction would eventually prevent 52.2 events per 1000 patients treated.
The estimated cost per YOLS, using a discount rate of $3 \%$, for male and female patients based on various ages at initiation of evolocumab treatment are presented in Table 2. For a population like that seen in the FOURIER study, treatment would save an average of 0.34 (95\% CI 0.27-0.41) life-years at a cost of $\$ 101899$ (95\% CI \$97 325-\$106 473), yielding a cost per YOLS of $\$ 299482$. In the sensitivity analysis using $1.5 \%$ discounting, the estimate was \$261 637 per YOLS, still well above established thresholds.

With advancing age, the benefits of treatment would decline (from 0.45 to 0.27 YOLSs for men and from 0.29 to 0.17 YOLSs for women) owing to the increasing risk of death from noncardiovascular causes and the smaller estimated remaining life expectancy. On the other hand, the costeffectiveness of treatment in terms of dollars per YOLS remained relatively stable across age groups (\$243 414$\$ 318335$ for men and \$382 796-\$528 009 for women), as older patients would receive fewer years of treatment.

Our probabilistic sensitivity analysis (Figure 1) estimated that to have a $50 \%$ probability of achieving a cost per YOLS below $\$ 50000$ and $\$ 100000$ would require annual drug costs below $\$ 1200$ and $\$ 2300$, respectively, assuming 3\% discounting. With a drug cost above $\$ 1800$ and $\$ 3000$, the probability of cost-effectiveness at thresholds of $\$ 50000$ and $\$ 100000$, respectively, was zero. In the sensitivity analysis using $1.5 \%$ discounting, the annual price of the drug would need to be

\begin{tabular}{|c|c|c|c|}
\hline \multirow[b]{2}{*}{ Event } & \multicolumn{3}{|c|}{ No. of events } \\
\hline & $\begin{array}{l}\text { Untreated } \\
\text { patients }\end{array}$ & $\begin{array}{l}\text { Treated } \\
\text { patients }\end{array}$ & $\begin{array}{l}\text { Events } \\
\text { prevented }\end{array}$ \\
\hline Recurrent myocardial infarction & 460.8 & 404.9 & 56.0 \\
\hline Cardiovascular death & 416.7 & 361.5 & 55.2 \\
\hline $\begin{array}{l}\text { New or recurrent myocardial } \\
\text { infarction }\end{array}$ & 484.2 & 429.9 & 54.3 \\
\hline $\begin{array}{l}\text { Cardiovascular death, myocardial } \\
\text { infarction (new or recurrent) or stroke }\end{array}$ & 752.7 & 700.7 & 51.9 \\
\hline Stroke or transient ischemic attack & 354.2 & 324.9 & 29.3 \\
\hline Fatal myocardial infarction & 149.2 & 120.1 & 29.2 \\
\hline Stroke & 293.8 & 266.3 & 27.5 \\
\hline Sudden death & 87.5 & 70.1 & 17.3 \\
\hline Stroke death & 96.4 & 83.8 & 12.5 \\
\hline Transient ischemic attack & 92.8 & 85.5 & 7.3 \\
\hline Newly diagnosed diabetes & 332.5 & 327.3 & 5.2 \\
\hline New myocardial infarction & 52.1 & 49.2 & 2.9 \\
\hline Angina pectoris & 41.5 & 38.7 & 2.8 \\
\hline Coronary insufficiency & 9.7 & 9.1 & 0.6 \\
\hline Congestive heart failure death & 84.9 & 88.5 & -3.6 \\
\hline Congestive heart failure & 107.5 & 111.2 & -3.7 \\
\hline Other death & 579.2 & 634.6 & -55.4 \\
\hline
\end{tabular}


Table 2: Average estimated years of life saved and associated costs by sex and age, discounted

\begin{tabular}{|c|c|c|c|c|c|}
\hline \multirow{2}{*}{$\begin{array}{l}\text { Sex; age at initiation } \\
\text { of evolocumab } \\
\text { treatment, yr }\end{array}$} & \multicolumn{2}{|c|}{ Years of treatment } & \multirow[b]{2}{*}{ YOLSs $(95 \% \mathrm{Cl})$} & \multirow{2}{*}{$\begin{array}{l}\text { Difference in lifetime cost } \\
\qquad(95 \% \mathrm{Cl})^{*}\end{array}$} & \multirow{2}{*}{$\begin{array}{l}\text { Cost pe } \\
\text { YOLS, } \$\end{array}$} \\
\hline & Untreated & Treated & & & \\
\hline \multicolumn{6}{|l|}{ Male } \\
\hline 45 & 18.6 & 19.1 & $0.45(0.37-0.53)$ & 144110 (115 628-172 593) & 318335 \\
\hline 55 & 15.6 & 16.0 & $0.42(0.34-0.51)$ & 120574 (97 031-144 117) & 284306 \\
\hline 65 & 12.1 & 12.5 & $0.36(0.29-0.44)$ & 93083 (76 276-109 890) & 257348 \\
\hline 75 & 8.6 & 8.8 & $0.27(0.23-0.31)$ & 65357 (54 354-76 359) & 243414 \\
\hline \multicolumn{6}{|l|}{ Female } \\
\hline 45 & 20.0 & 20.3 & $0.29(0.20-0.37)$ & 151539 (120 778-182 299) & 528009 \\
\hline 55 & 16.9 & 17.2 & $0.29(0.20-0.37)$ & 128236 (102 937-153 535) & 448221 \\
\hline 65 & 13.3 & 13.6 & $0.26(0.20-0.33)$ & 99871 (82 313-117 430) & 382796 \\
\hline 75 & 9.7 & 9.9 & $0.17(0.12-0.21)$ & 71607 (60 198-83 016) & 422710 \\
\hline Overall† & 13.3 & 13.6 & $0.34(0.27-0.41)$ & 101899 (97 325-106 473) & 299482 \\
\hline $\begin{array}{l}\text { Note: } \mathrm{Cl}=\text { confidence inte } \\
{ }^{*} \text { In current Canadian doll } \\
\text { †Weighted for the age an }\end{array}$ & $\begin{array}{l}\text { OLS = year } \\
\text { ( Methods). } \\
\text { listributions }\end{array}$ & $\begin{array}{l}\text { saved. } \\
\mathrm{n} \text { the FO }\end{array}$ & trial. ${ }^{2}$ & & \\
\hline
\end{tabular}

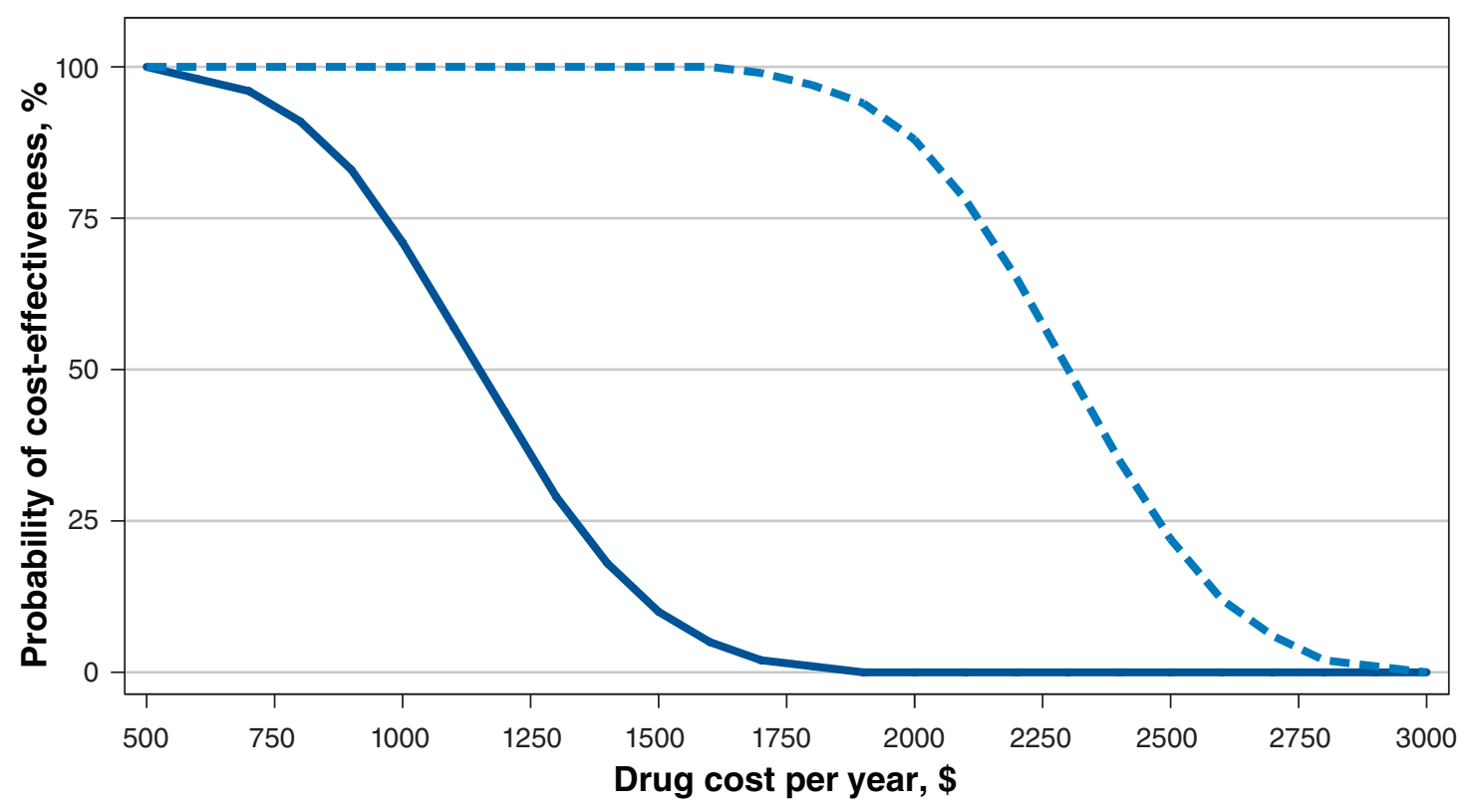

Cost threshold per year of life saved $\$ 50000$

브뭉 $\$ 100000$

Figure 1: Cost-effectiveness acceptability curves showing, as a function of the annual drug cost, the proportion of 1000 simulated results that would give, for a specified willingness-to-pay amount, a positive incremental net benefit. 
below $\$ 1408$ and $\$ 2847$ to have any chance at being costeffective at thresholds of $\$ 50000$ and $\$ 100000$, respectively.

\section{Interpretation}

No cardiovascular or total mortality benefit was seen in the FOURIER trial; nonetheless, there is a possibility that lowering of LDL levels by PCSK9 inhibitors such as evolocumab may reduce mortality over time. However, despite this critical assumption, evolocumab is unlikely to be cost-effective for secondary prevention at current Canadian prices.

In contrast, in a similar analysis for secondary prevention with statins in patients at high risk performed nearly 20 years ago, ${ }^{8}$ the cost per YOLS was estimated at $\$ 6522-$ $\$ 12402$ in 2017 Canadian dollars. This statin analysis was based on a lower-potency statin that was still patented; hence, a similar analysis performed today with high-potency generic statins would give even more favourable results. In a similar analysis involving the subsequent addition of ezetimibe to a statin, Kazi and colleagues ${ }^{19}$ estimated a cost of US\$199 000 per quality-adjusted life-year (QALY) compared to a statin alone. These results underscore the challenges associated with identifying cost-effective lipid therapies when compared with the low cost and demonstrated effectiveness of statins.

Our results differ from those of other recently published analyses of the FOURIER study in that they involve Canadian cost estimates for both the drug and medical costs associated with cardiovascular disease and in that we present YOLSs, which provide a lower cost estimate than QALYs. Arrieta and colleagues ${ }^{20}$ published an updated model estimating a cost of US\$337 729 per QALY and requiring a discount to $\$ 5459$ per year to reach US\$100 000 per QALY. Similarly, Kazi and colleagues ${ }^{19}$ updated earlier estimates to US\$339000 per QALY, requiring a discount to US\$4215 to achieve US\$100 000 per QALY when comparing to the addition of ezetimibe. Importantly, those authors also confirmed that the estimated cost per life-year saved was lower than the cost per QALY. Furthermore, they showed that, if no mortality benefit were realized, the cost per QALY would correspondingly increase to US\$1 795000 . Fonarow and colleagues $^{21}$ estimated US\$268 637 per QALY gained, with a threshold of US\$150 000 per QALY being met at an annual price of US\$9669. Thus, including our study, all the major studies conducted after the results of the FOURIER trial became available have shown that evolocumab is not costeffective for secondary prevention at current pricing in either the US or the Canadian context.

\section{Limitations}

The principal limitation of our study is that the results are based on a model not specifically developed for PCSK9 inhibitors. The assumption that cardiovascular and all-cause mortality will be lowered by PCSK9 inhibitors in a manner similar to the results observed for statins remains theoretical. There are several important examples of drugs that lower LDL levels but have not been shown to reduce mortality. ${ }^{22-24}$
Thus, our results likely represent a best-case scenario given the data currently available. In addition, we modelled a secondary prevention population like that seen in the FOURIER trial. It is possible that a more highly selected population could be identified for whom the drug might offer more incremental benefit to offset the costs. Finally, our approach is also limited by not including the potential cost of adverse effects that may be recognized only after a new medication has been used over the longer term. For instance, cognitive impairment remains a concern with extremely low LDL levels, and a 2-year trial may be insufficient to address this issue. $^{25}$

These analyses focused only on evolocumab as observed in the FOURIER trial. We cannot generalize about the use of evolocumab for other clinical indications or the entire class in terms of cost-effectiveness. Finally, we also assume that the drug will maintain efficacy over the duration of treatment; however, development of another member of the class has been stopped because of immune reactions against the monoclonal antibody, ${ }^{26}$ and antidrug antibodies to others within the class have been reported. ${ }^{27}$

\section{Conclusion}

Using a well-validated model calibrated specifically for the cardiovascular event reduction observed in the FOURIER study, we found that, at current pricing, evolocumab is unlikely to be cost-effective in Canada for preventing death in secondary prevention as per the published results of the FOURIER trial. With evolving clinical trial data, future analyses will need to reevaluate whether these medications are sufficiently effective and cost-effective to become the standard of care for specific groups of Canadian patients.

\section{References}

1. Heart disease in Canada. Ottawa: Public Health Agency of Canada; 2017. Available: https://www.canada.ca/content/dam/phac-aspc/documents/services/ publications/diseases-conditions/heart-disease-maladies-coeur-eng.pdf (accessed 2017 May 10).

2. Sabatine MS, Giugliano RP, Keech AC, et al.; FOURIER Steering Committee and Investigators. Evolocumab and clinical outcomes in patients with cardiovascular disease. N Engl 7 Med 2017;376:1713-22.

3. Dixon DL, Buckley LF, Trankle CR, et al. Clinical utility of evolocumab in the management of hyperlipidemia: patient selection and follow-up. Drug Des Devel Ther 2017;11:2121-9.

4. Grover SA, Paquet S, Levinton C, et al. Estimating the benefits of modifying risk factors of cardiovascular disease: a comparison of primary vs secondary prevention. Arch Intern Med 1998;158:655-62.

5. Grover SA, Kaouache M, Rempel P, et al. Years of life lost and healthy lifeyears lost from diabetes and cardiovascular disease in overweight and obese people: a modelling study. Lancet Diabetes Endocrinol 2015;3:114-22.

6. Grover SA, Coupal L, Gilmore N, et al. Impact of dyslipidemia associated with highly active antiretroviral therapy (HAART) on cardiovascular risk and life expectancy. Am 7 Cardiol 2005;95:586-91.

7. Grover SA, Coupal L, Kaouache M, et al. Preventing cardiovascular disease among Canadians: What are the potential benefits of treating hypertension or dyslipidemia? Can 7 Cardiol 2007;23:467-73.

8. Grover SA, Coupal L, Paquet S, et al. Cost-effectiveness of 3-hydroxy3-methylglutaryl-coenzyme A reductase inhibitors in the secondary prevention of cardiovascular disease: forecasting the incremental benefits of preventing coronary and cerebrovascular events. Arch Intern Med 1999;159:593-600.

9. Grover SA, Coupal L, Zowall H, et al. Cost-effectiveness of treating hyperlipidemia in the presence of diabetes: Who should be treated? Circulation 2000, 102:722-7.

10. The Lipid Research Clinics Coronary Primary Prevention Trial results. I. Reduction in incidence of coronary heart disease. FAMA 1984;251:351-64.

11. Kannel W, Wolf P, Garrison R. The Framingham Study: an epidemiological investigation of cardiovascular disease. Section 35: survival following initial cardio- 
vascular events: 30 year follow-up. Springfield (VA): National Technical Information Service; 1988.

12. Randomised trial of cholesterol lowering in 4444 patients with coronary heart disease: the Scandinavian Simvastatin Survival Study (4S). Lancet 1994;344: $1383-9$.

13. Manninen V, Elo MO, Frick MH, et al. Lipid alterations and decline in the incidence of coronary heart disease in the Helsinki Heart Study. FAMA 1988; 260:641-51.

14. Shepherd J, Cobbe SM, Ford I, et al. Prevention of coronary heart disease with pravastatin in men with hypercholesterolemia. N Engl f Med 1995;333: 1301-7.

15. Buchwald H, Varco RL, Matts JP, et al. Effect of partial ileal bypass surgery on mortality and morbidity from coronary heart disease in patients with hypercholesterolemia: report of the Program on the Surgical Control of the Hyperlipidemias (POSCH). N Engl 7 Med 1990;323:946-55.

16. PCSK9 inhibitor monoclonal antibodies for the treatment of hypercholesterolemia. Ottawa: Canadian Agency for Drugs and Technologies in Health; 2015. Available: https://www.cadth.ca/dv/ieht/pcsk9-inhibitor-monoclonal-antibodies -treatment-hypercholesterolemia (accessed 2017 May 10).

17. Sanders GD, Neumann PJ, Basu A, et al. Recommendations for conduct, methodological practices, and reporting of cost-effectiveness analyses: Second Panel on Cost-Effectiveness in Health and Medicine. 7AMA 2016;316: 1093-103.

18. Guidelines for the economic evaluation of health technologies: Canada. 4th ed. Ottawa: Canadian Agency for Drugs and Technologies in Health; 2017. Available: https:// www.cadth.ca/about-cadth/how-we-do-it/methods-and-guidelines/guidelines -for-the-economic-evaluation-of-health-technologies-canada (accessed 2017 Dec. 1).

19. Kazi DS, Penko J, Coxson PG, et al. Updated cost-effectiveness analysis of PCSK9 inhibitors based on the results of the FOURIER trial. FAMA 2017; 318:748-50.

20. Arrieta A, Hong JC, Khera R, et al. Updated cost-effectiveness assessments of PCSK9 inhibitors from the perspectives of the health system and private payers: insights derived from the FOURIER trial. FAMA Cardiol 2017;2:1369-74.

21. Fonarow GC, Keech AC, Pedersen TR, et al. Cost-effectiveness of evolocumab therapy for reducing cardiovascular events in patients with atherosclerotic cardiovascular disease. FAMA Cardiol 2017;2:1069-78.

22. Fei Y, Guyatt GH, Alexander PE, et al. Addition of ezetimibe to statins for patients at high cardiovascular risk: systematic review of patient-important outcomes. $\mathcal{F}$ Eval Clin Pract 2017 Jan. 16 [Epub ahead of print]. doi:10.1111/ jep.12663.

23. Wang D, Liu B, Tao W, et al. Fibrates for secondary prevention of cardiovascular disease and stroke. Cochrane Database Syst Rev 2015;(10):CD009580.

24. Schwartz GG, Olsson AG, Abt M, et al. Effects of dalcetrapib in patients with a recent acute coronary syndrome. N Engl 7 Med 2012;367:2089-99.

25. Lipinski MJ, Benedetto U, Escarcega RO, et al. The impact of proprotein convertase subtilisin-kexin type 9 serine protease inhibitors on lipid levels and outcomes in patients with primary hypercholesterolaemia: a network meta-analysis. Eur Heart 7 2016;37:536-45.

26. Ridker PM, Tardif JC, Amarenco P, et al. Lipid-reduction variability and antidrug-antibody formation with bococizumab. N Engl f Med 2017;376: 1517-26.

27. Roth EM, Goldberg AC, Catapano AL, et al. Antidrug antibodies in patients treated with alirocumab. N Engl 7 Med 2017;376:1589-90.

Affiliations: Division of General Internal Medicine (Lee, Grover), Department of Medicine, McGill University; Clinical Practice Assessment Unit (Lee), McGill University Health Centre, Montréal, Que.; Department of General Sciences (Kaouache), Prince Sultan University, Riyadh, Kingdom of Saudi Arabia; Centre for the Analysis of CostEffective Care (Grover), Montreal General Hospital, Montréal, Que.

Contributors: Steven Grover acquired the data. Mohammed Kaouache and Steven Grover analyzed the data, and Todd Lee and Steven Grover interpreted the data. Todd Lee drafted the manuscript. All of the authors contributed substantially to the study conception and design, revised the manuscript critically for important intellectual content, gave final approval of the version to be published and agreed to be accountable for all aspects of the work.

Funding: This study was supported by grant GIR-86935 from the Canadian Institutes of Health Research.

Supplemental information: For reviewer comments and the original submission of this manuscript, please see www.cmajopen.ca/content/6/2/ E162/suppl/DC1. 